\title{
Sistemas productivos locales y encadenamientos productivos a favor del desarrollo local: El sistema productivo local agropecuario del destino turístico Gibara en holguín
}

\author{
Local production systems and production chains in favor of local \\ development: The local agricultural production system of the Gibara \\ tourist destination in Holguín
}

MsC. Prof. Aux. Merlinda Clarke Bloomfield. ${ }^{1}$, Dra.C Ana Gloria Madruga Torres. ${ }^{2}$

Ing. José Luis Carballosa Ramírez ${ }^{3}$ \& Ing. Crecencio Ramón Rodríguez Galindo ${ }^{4}$

\begin{abstract}
The Strategic Axis Productive transformation and international insertion contained in the National Plan until 2030 emphasizes the need for the development of strategic sectors that have a long-term impact on the development of the economy and society; Tourism was identified as a catalyst for territorial economies, which has a specific expression at the local level, so it must be part of development on a territorial scale based on productive chains as one of the ways to manage such development. The objective of the present investigation was to diagnose the Agricultural Productive Local System (SPL) of the Gibara municipality and its conditions to contribute to the development strategy of the municipality and the tourism sector, as well as the identification of the production chains present in the SPL studied; To do this, a procedure was developed and applied based on the analysis of the economic structure of the municipality that contributes to promoting the development of tourism as a strategic sector in the territorial economic structure. The applied procedure allowed to create the necessary organizational conditions for the development of the investigation from establishing the basic input information for the diagnosis, obtaining as results the determination of the current situation of the

\footnotetext{
${ }^{1}$ Profesora Auxiliar del Departamento de Economía de la Universidad de Holguín; Master en Ciencias, email: merlinda@uho.edu.cu

2 Profesora Titular del Departamento de Desarrollo Local de la Universidad de Holguín, Doctora en Ciencias Económicas, amadruga@uho.edu.cu

${ }^{3}$ Graduado de Ingeniero Industrial, 2019

${ }^{4}$ Director de la UEB Productora y Comercializadora de Conservas de Frutas y Vegetales, Holguín.
} 
Agricultural SPL, the identification of 31 production chains and the diagnosis, design and implementation of the guava production chain.

Key Words: Local Development, Productive Links, Local Productive Systems, Local Agricultural Productive System.

\section{Resumen}

El Eje Estratégico Transformación productiva e inserción internacional contenido en el Plan de la Nación hasta el 2030 enfatiza en la necesidad del desarrollo de sectores estratégicos que a largo plazo impacten en el desarrollo de la economía y la sociedad; el turismo fue identificado como dinamizador de las economías territoriales, lo cual tiene una expresión concreta en el nivel local, por lo que debe formar parte del desarrollo a escala territorial a partir de los encadenamientos productivos como una de las vías para gestionar tal desarrollo. La presente investigación tuvo como objetivo diagnosticar el Sistema Productivo Local (SPL) Agropecuario del municipio Gibara y sus condiciones para tributar a la estrategia de desarrollo del municipio y el sector del turismo, así como la identificación de las cadenas productivas presentes en el SPL estudiado; para ello se elaboró y aplicó un procedimiento que parte del análisis de la estructura económica del municipio que contribuya a potenciar el desarrollo del turismo como sector estratégico en la estructura económica territorial. El procedimiento aplicado permitió crear las condiciones organizativas necesarias para el desarrollo de la investigación a partir de establecer la información básica de entrada para el diagnóstico obteniendo como resultados la determinación de la situación actual del SPL Agropecuario, la identificación de 31 cadenas productivas y el diagnóstico, diseño e implementación de la cadena productiva de la guayaba.

Palabras Clave: Desarrollo Local, Encadenamientos Productivos, Sistemas Productivos Locales, Sistema Productivo Local Agropecuario.

\section{Introducción}

La concentración geográfica de empresas pertenecientes a un sector de actividad económica o aglomeración productiva (AP), se presenta en la actualidad como un elemento clave en la gestión del desarrollo local (DL) Vázquez, (2006, 2007 y 2009), porque no solo contribuyen al mejoramiento del desempeño de las empresas e instituciones que las integran, sino también, al de los territorios donde se encuentran localizadas como consecuencia de los procesos de articulación entre estas y el efecto sinérgico que genera, por lo que el estudio y fomento de las formas de organización de la producción sustentadas en las aglomeraciones productivas y en particular los Sistemas Productivos Locales (SPL), constituyen una alternativa estratégica que favorece de manera significativa al logro de la competitividad y el desarrollo económico de las regiones en que estos progresan.

Un componente esencial de los SPL como AP y comercialización lo constituyen las cadenas productivas (CP), enfoque que surge en la década de los 50 abordada por los profesores Davis y Goldberg (1957), los cuales por vez primera presentan un estudio que 
recoge una visión sistémica de la agricultura, en el que se refleja la interrelación entre todos sus procesos para hacer llegar a los consumidores las ofertas de productos de la agricultura, mecanismos de articulación productiva encargados de generar los vínculos intra e intersectorial necesarios para que se produzca la transformación de la estructura económica del territorio.

De la formación de SPL como factor del Desarrollo Local (DL) en Cuba se obtendrá una oportunidad para reestructurar la actividad productiva partiendo de las aglomeraciones o concentraciones sectoriales existentes en los territorios, las cuales se irán transformando por los encadenamientos que se puedan crear, Madruga y González (2011), consideran que no obstante, el funcionamiento de estas han estado limitadas por la insuficiente articulación productiva que se expresa en la existencia de brechas que se producen entre las organizaciones de un mismo sector y entre sectores, que son consecuencia de causas asociadas a la gestión de los factores que determinan el nivel de articulación productiva intra e inter Aglomeraciones Productivas (AP) del territorio, como manifestación del proceso de organización de la producción con enfoque territorial, Madruga Torres, (2016), lo que ocasiona que no se aprovechen las ventajas competitivas y comparativas de los territorios o localidades, dificultando la generación de desarrollo tanto económico como social. Por lo que constituyen una alternativa viable, debido a que no solo establecen un modo de organización empresarial que surge de la estructura productiva existente en un territorio, sino que responde a un conjunto de problemáticas presentes en los territorios a partir del enfoque sectorial que prima en los procesos de desarrollo, proceso que no se puede concebir sin tener en cuenta el mejoramiento del nivel y calidad de vida de la población.

Por ello el país ha dado prioridad al enfoque de gestión de desarrollo desde lo local, con base en las políticas planteadas en los Documentos aprobados en el VII Congreso del Partido en los que el DL se identifica como una vía fundamental de desarrollo que tiene como fin aprovechar con eficiencia los recursos de que dispone cada territorio y de esta forma potenciar la economía de los municipios; Lineamientos de la Política Económica y Social del Partido (2017, pp. 6, 29), 17, y 165, políticas refrendadas en el Plan Nacional hasta 2030, en el Eje Estratégico: Transformación productiva e inserción internacional el objetivo específico 5, todo ello en correspondencia con el objetivo 8 del documento: Transformar nuestro mundo: La Agenda 2030 para el Desarrollo Sostenible (2015, p. 22) aprobado por la Organización de Naciones Unidas, por lo que el DL se asume como "un proceso esencialmente endógeno, sustentado en la correcta utilización por parte de los actores locales de los recursos de diverso tipo que se encuentran insertados dentro de los límites de un determinado territorio. Es una resultante directa de la capacidad de los actores y de la sociedad local para estructurase y movilizarse con base en sus potencialidades y poner en función de su localidad los recursos exógenos en estrecha armonía con sus recursos endógenos que la localidad es capaz de generar“, Indicaciones Metodológicas para la Elaboración del Plan 2020, Anexo a la Resolución No. 145/2019.

Ahora bien a este proceso, no es ajena la provincia Holguín quien impulsa el DL como una estrategia e instrumento para garantizar no sólo crecimientos económicos, sino que 
busca maneras prácticas de fortalecer las estructuras de los municipios y sus estrategias de desarrollo apoyado en el Programa Nacional de Desarrollo Local (Consejo de Administración Provincial(CAP), p.21); provincia que, en resultados de estudios anteriores Diagnóstico Prospectivo Estratégico del municipio de Gibara (Mayán, 2017), se demostró la existencia de AP como base para la conformación de SPLs y posibles cadenas productivas $(\mathrm{CP})$; en los municipios de la provincia se pudo apreciar además la existencia de potencialidades para fomentar el desarrollo de sectores como la agricultura que se reconoce como actividad económica fundamental en 12 de los 14 municipios de la provincia y el turismo en de los 14 municipios aunque en todos se cuenta con potencialidades para su explotación, pero las relaciones de articulación entre los productores y la industria son insuficientes, así como el aprovechamiento de las potencialidades de producción que permita el uso eficiente de las capacidades instaladas en las industrias que permita proveer de productos frescos de la agricultura y productos terminados cuya materia prima proviene de este sector.

Ello se manifiesta en Gibara, municipio objeto de estudio práctico de la investigación, ¿por lo que en la investigación se busca cómo favorecer el aprovechamiento de las potencialidades del municipio a partir de la aplicación del enfoque de SPL, la misma tiene como objetivo caracterizar la situación del SPL agropecuario en el municipio Gibara para favorezca la identificación de relaciones de articulación con el sector del turismo a partir de las CP, como base para el diseño e implementación de proyectos que atenúen o eliminen los vacíos en las relaciones de articulación entre los diferentes actores que intervienen en las mismas; lo que se constituye en base importante para el logro de la eficiencia en las relaciones de articulación entre sus eslabones en la conformación del SPL agropecuario del municipio, que contribuya a una acertada orientación de las estrategias del territorio hacia el futuro que se quiere alcanzar, por lo que a partir del procedimiento elaborado y aplicado se ejemplifica con el caso de la cadena productiva de la guayaba.

\section{Desarrollo}

En investigaciones realizadas y en su tesis doctoral Madruga Torres, 2015 realiza una profunda revisión de la literatura nacional e internacional sobre los SPL en las que se aprecian diversos aportes para identificar y diagnosticar sistemas productivos locales, destacan (Marshall (1890); Hirschman (1958); Bagnasco (1977); Brusco (1982); Pyke, Becattini y Segenberger (1990); Boisier (1997); Porter (1998 y 2003); Fukuyama (1995); Cassiolato y Szapiro (2002); European Commission (2002); Rosenfeld (2002); Caravaca, et al (2003); Negrín (2004); Andersson, Schawaag-Seger, Sorvik y Hansson (2004); Becattini (1987, 1992, 2006) y González Fontes (2011) entre otros; aportes teóricos que se consideran significativos y que ofrecen instrumentos para su identificación; sin embargo, en ninguno se aprecia cómo identificar los vacíos que se dan en las relaciones de articulación intra e intersectoriales Madruga, (2011), la que destaca como resultado del análisis que, a pesar de sus aportaciones al pensamiento teórico, el instrumental sobre 
el que se sustentan fueron concebidas para economías que funcionan bajo la lógica del mercado, de ahí que aplicarlos acríticamente pudiera no aportar los resultados esperados en las condiciones cubanas.

Además, profundiza en estudios realizados por autores cubanos que se orientan al intento de adaptar dichas metodologías al contexto nacional; Monreal, (2002); Negrín, (2004); Marquetti, (2006); González, (2011), y concluye que las mismas presentan carencia metodológica, al no aportar vías para la identificación de las causas que generan los vacíos en las relaciones de articulación y que pueden frenar el DL.

Tal es el caso de Naclerio (2008) que se refiere a los SPL “... como empresas asociadas y localizadas en un territorio, que se asocian en la realización de actividades para lograr objetivos comunes, que pueden ser del mismo sector o de una cadena de valor”. Y agrega que "La idea fuerza detrás de los SPL es que la construcción de capacidades productivas no depende del desempeño aislado de cada uno de sus actores“ (p. 2, 3).

Por otra parte, (Rendón Acevedo \& Forero Muñoz, 2014) plantean que "...pueden definirse como estructuras o modos de organización empresariales orientados a la producción y comercialización de un determinado bien o servicio. Pero estas estructuras encierran una complejidad que es difícil de lograr en una definición corta, dado el vínculo que estos deben tener con el territorio que los sustenta."(p. 77).

Las diferentes aportaciones científicas de los autores nacionales e internacionales, permitieron a Madruga Torres $(2011,2014,2015)$, marcar una pauta para concebir el concepto de SPL para el caso cubano, a partir del reconocimiento de las características de los procesos naturales de obtención de valor, los que son válidos para cualquier tipo de sistema económico. En este ámbito defiende el criterio de que los SPL en Cuba, están definidos por: 1) la dimensión territorial; 2) la diversidad de actividades y actores interrelacionados; 3) la innovación; 4) el conocimiento tácito y 5) la coordinación del sistema, (Madruga Torres, 2014, p.6)

Esas características unidas a la identificación de un conjunto de insuficiencias teóricometodológicas (Madruga Torres y González Fontes, 2011, p.5) en la práctica del desarrollo local en Cuba en general y de los SPL en particular le permitieron un acercamiento hacia la definición de SPL para el caso de cubano a partir de las variables consideradas por estos autores y asume como concepto de SPL:

“el espacio de interacción definido por las relaciones entre empresas con funciones específicas en cada fase del proceso de producción con presencia de especialización en las relaciones productivas, que se pueden manifestar mediante la generación de vínculos territoriales entre estas instituciones, existiendo una interacción continua entre la actividad económica, cultural, social y política; presentando una estructura organizativa que dirige las funciones en el espacio definido, donde existe una complementación de funciones entre los diferentes agentes locales orientados a fortalecer la capacidad de conocer, aprender e innovar, convirtiéndolo en un núcleo fundamental de la dinámica de una economía local“" (Madruga Torres, 2015. p.6-17) 


\section{Sistema productivo local agrícola}

Como se aprecia en la literatura, los SPL se suelen concebir como un modelo de desarrollo territorial endógeno, de manera que muchos de los factores que determinan su formación y desarrollo son controlados por los actores del territorio, sin embargo, en el análisis de la literatura sobre los SPL agrícolas se ha podido apreciar que el tema es ampliamente tratado para el sector industrial en lo fundamental desde el pasado siglo según refieren Rendón Acevedo y Forero Muños (2014) a partir de lo cual se han aportado un sinnúmero de estudios teóricos y resultados empíricos sobre la temática; pero cuando se analiza el sector agropecuario contrariamente al peso que este tiene en las localidades tanto de Cuba como en otros países, los estudios teóricos y las evidencias empíricas revelan su amplio uso en países como Italia (Becattini, Brusco y Sforzi), España, Alemania, Francia, Suecia y Reino Unido, países que en las décadas de 1970 y 1980 gran parte de su economía entró en recesión, con fuerte deterioro del empleo y aumento de la incertidumbre, no obstante mostraron una gran capacidad de adaptación al nuevo entorno económico, llegando incluso a lograr fases de crecimiento estables a partir del desarrollo que tenían en los SPL o Distritos Industriales.

En América se encuentran distritos y SPL que han impulsado el crecimiento económico en localidades de Estados Unidos (Silicon Valley), México (Jalisco y Guanajuato), Brasil (Sao Paulo y Porto Alegre), etc. Asociados en lo fundamental a la actividad industrial, de igual manera en la actividad agropecuaria los estudios y casos de SPL no son tan abundantes como en el sector industrial, siendo más profusa la información sobre los encadenamientos productivos, en lo fundamental herramientas para su conformación en países de América Latina como Argentina, Brasil, Chile, Colombia, el Salvador, Guatemala, etc., como estrategia de desarrollo regional y en este mismo sentido abordado por autores como, Domenech (2004), Alburquerque (2004), García y Noguera (2004), Naclerio (2008), Ferrán et.al, (2009), Jáuregui (2012), Vergaray (2013), Rendón y Acevedo (2014) entre otros.

De aquí que no se encuentre una teoría acabada acerca de los SPL agrícolas, aunque sí se reconoce su importancia con base en la teoría de la organización y localización de las actividades económicas en el espacio entre ellas: la teoría de la localización agraria de Johan Vhon Thünen (1826), reconocido como padre de las teorías de la localización quien se proyecta del espacio rural al espacio urbano; hasta la etapa actual en que se aborda el espacio desde una nueva perspectiva, es decir la perspectiva territorial, en la cual se considera el espacio como un factor esencial en la comprensión de los procesos de crecimiento y desarrollo, por tanto se trata entonces de un nuevo planteamiento, no de una nueva teoría, ya que el desarrollo endógeno que es la base en que se sustenta este planteamiento, aún no cuenta con un sistema conceptual, leyes y categorías bien definidas y unificadas.

Por lo que el sistema productivo local agrario se puede concebir como un conjunto de elementos complejos, organizados, relacionados dinámicamente, articulados, entre sí e interdependientes, para cumplir objetivos comunes; con este enfoque, se ha de observar 
la agricultura como un fenómeno muy complejo en el cual un gran número de elementos interactúan, organizados en una estructura determinada con la finalidad de producir alimentos y otros productos que el hombre y la sociedad necesitan, por tanto este como unidad, influye y es influenciado por otros sistemas similares locales (vecinos o no), permitiendo un efecto retroalimentador, en mayor o menor grado, con los niveles jerárquicos superiores nacionales, provinciales y municipales e incluso a nivel de Consejo Popular, influencia que se puede considerar multifactorial. Es decir que en una misma estructura y en una misma especie de producción (Carne: vacunos, ovejas, cerdos, etc. Cultivos varios: frutas, viandas, hortalizas y granos) se pueden tener muchos sistemas de producción distintos.

El análisis de la literatura nacional e internacional aporta elementos acerca de la necesidad del estudio de los SPL, pero todos coinciden en su razonamiento a partir del aporte de los distritos industriales y lo adaptan a la actividad agroindustrial observándose una amplia diversidad de criterios recientes bajo diversas acepciones entre ellas agroindustria, agropecuario, agrícola: Jouvé (1978), Vissac (1979), Pengue (2005), Dogliotti (2007), Piñeiro (2008), Echave (2009), por su parte refiere Bocco (2013) y organizaciones como PNUD (2006), ONUDI (2006),SEBRAE (2005), etc. Quienes demuestran que no es un concepto acabado a pesar de la existencia de una amplia literatura en torno a las CP agrícolas y pecuarias, de ahí que en correspondencia con el planteamiento de Madruga (2015), a partir de la identificación con la aplicación de Técnicas de Análisis Regional (TAR) y herramientas empíricas, en que se identifique la agricultura como actividad económica fundamental del territorio o localidad objeto de estudio, se asume en la presente investigación que el SPL Agrícola para las condiciones de Cuba se refiere a: un conjunto de empresas y formas productivas históricamente constituidas y duraderas localizadas en un mismo territorio, en correspondencia con las condiciones de un espacio determinado y donde se establecen relaciones de articulación entre los actores y con otros actores locales con el fin de producir productos que satisfagan necesidades.

El SPL Agrícola puede estar constituido a su vez por subsistemas en correspondencia con los subsectores existentes en la estructura económica territorial, entre los que destacan los Sistemas agrícolas, Pecuarios, Silvícolas, Caza, Pesca; compuestos a su vez por CP, cuyo funcionamiento en forma de red organizada posibilita la conformación del sistema en sí, por tanto (Naclerio, 2010, p. 16) "La complementariedad institucional entre actores tales como organismos públicos de investigación, universidades y empresas, deviene un foco sustancial que motoriza el interés tanto de la academia como de la gestión“

En la formación de SPLs Agrícolas como mecanismo en la gestión del DL, reviste gran relevancia para los territorios, al menos en su fase más incipiente, el enfoque o concepción de cadenas productivas a partir de las actividades económicas más importantes para el territorio, por lo que la conformación de SPL Agrícolas en un espacio territorial delimitado tiene un fuerte impacto positivo en la competitividad de las empresas que lo integran y contribuye a mejorar las condiciones económicas y sociales del entorno en el que se desarrolla, por lo que según Aznar(Aznar, 2011, p. 7) "en un contexto internacional de creciente competencia y concentración de la demanda, constituyen una forma eficaz 
de integración en el complejo mercado mundial y un importante elemento de competitividad"

Por lo que un componente esencial de los SPL son las CP; al decir de Forero para que exista un SPL debe estar presente una intención coordinada de producción y comercialización, y esto implica un trabajo conjunto entre empresas, el cual va desde los primeros tratamientos de una materia prima, hasta la venta del producto final. Cabe destacar que la existencia de una cadena productiva no implica necesariamente la existencia de un sistema productivo local, pero este no es concebible si dentro de él no se identifican cadenas productivas. Si se analiza a fondo, se encuentra que las cadenas productivas se componen de redes, orientadas hacia la producción y puesta en el mercado de un producto" (Forero, 2013) citado por (Rendón Acevedo y Forero Muños, 2014, p. 81)

\section{Diagnóstico del spl agropecuario del municipio gibara}

Para la realización del diagnóstico del SPL se tomó como base el planteamiento teórico del procedimiento elaborado (Anexo 1) por el grupo de proyecto "Diagnóstico de las cadenas productivas como herramienta básica para la formulación de políticas públicas", tomando como fuente primaria el instrumento para la identificación de Aglomeraciones productivas como base para los Sistemas Productivos Locales, elaborado por Madruga Torres, 2015 en su tesis doctoral, el que sirvió de base para orientar la presente investigación, utilizando indicadores económicos e información estadística en las unidades productivas, empleo, producción y otras variables en este caso agrícolas, relevantes para el análisis.

\section{Caracterización del municipio gibara}

Gibara fue fundado el 16 de enero de 1817, y desde 1975 es la cabecera del municipio del mismo nombre, cuenta con una extensión territorial de $618.82 \mathrm{Km}^{2}$, con $37 \mathrm{Km}$ de costa. Se encuentra ubicado geográficamente en la porción Noroeste de la Provincia Holguín entre las coordenadas $57^{\circ}$ Latitud Norte y $80^{\circ}$ Longitud Oeste, limita al Norte con el Océano Atlántico, al Sur con el Municipio de Holguín, al Este con el de Rafael Freyre, al Oeste con la provincia de Las Tunas y Municipio Calixto García. Su principal accidente costero es La Bahía de Gibara. Su red hidrográfica es de baja intensidad y sus ríos principales son Cacoyogüín, Gibara y Mano, posee 2 Presas y 33 micropresas.

El sistema de asentamientos poblacionales del municipio está formado por un total de 122 asentamientos, de ellos 5 urbanos y 117 rurales. Lo estructuran 10 Consejos Populares 7 mixtos (Gibara 1 y 2, Velasco 1 y 2, Floro Pérez, Bocas y Uñas) y 3 rurales (Cañada de Melones, Arroyo Seco y Caletones). Cuenta además con 103 circunscripciones de ellas 37 urbanas, 7 semiurbanas y 59 rurales. Gibara como ciudad tiene un área total aproximada de 190 ha, con una población de 18439 habitantes de ellos 8749 mujeres y 9690 hombres.

Su población total alcanza los 72056 habitantes, de ellos 36977 son de sexo masculino y 36079 femenino, con una densidad poblacional de $114 \mathrm{hab} / \mathrm{Km}^{2}$ y por su parte Gibara 
como ciudad tiene un área total aproximada de 190 ha, con una población de 18439 habitantes de ellos 8749 mujeres y 9690 hombres.

La ciudad se encuentra estructurada a partir de un área central que incluye el centro comercial y cultural alrededor del cual se localizan las áreas residenciales, las que constituyen el 68,7 \% del área total urbana. Las instalaciones de producción se encuentran dispersas sin conformar zonas; posee un centro histórico con grandes valores arquitectónicos y urbanísticos, razón por la cual fue declarada Monumento Nacional y en el cual se celebra cada año el Festival Internacional de Cine.

Las actividades fundamentales de la economía del municipio son la rama ligera (Empresa Hilandería" Hinejiro Asanuma"), agroalimentaria (Productora y Distribuidora de Alimentos de Gibara), construcción de maquinarias y equipos (Astilleros Norte Oriental, Astinor), energética ( 2 parques eólicos), tabaco (5 fábricas) y la producción de materiales de construcción (Empresa Constructora de subordinación local); estando presentes 6 de las 10 ramas que rigen la política industrial en Cuba reconocidas por el Ministerio de Economía y Planificación. La producción mercantil del municipio representa un $0.85 \%$ de la producción de la provincia lo que indica que la industria está deprimida fundamentalmente por el déficit de materia prima ya que existe capacidad instalada.

El turismo a pesar no estar considerado dentro de la estructura económica territorial en la información estadística que aporta la ONEi, es una de las actividades productivas fundamentales del municipio y sin dudas un sustento económico esencial para el territorio; las potencialidades que avalaron a Gibara y el territorio aledaño como destino turístico en 2017, fue evaluado en un radio no mayor de $20 \mathrm{~km}$, donde se concentran un conjunto de atractivos unidos a la ciudad. El municipio cuenta con 4 hoteles de categoría Encanto (E), pertenecientes a Compañía Hoteles de la cadena Iberostar: el Hotel Ordoño, el Hotel Plaza Colón, el Arsenita, el Hostal Buena Vista y en construcción el Hotel Bahía del Almirante. Es el único territorio en la provincia con tales características y sus espacios turísticos están representados por:

- Espacio Turismo Urbano

- Espacio Turismo de Naturaleza

- Espacio Turismo Náutico

- Espacio Turismo Rural

Entre los atractivos turísticos con los que cuenta el municipio se distinguen: sus playas, la bahía, sus cuevas y paisajes naturales, la belleza de sus fondos marinos, el casco histórico, las edificaciones, etc.

Se encuentran también en el espacio gibareño riquezas naturales y socioculturales que forman parte de los atractivos para el turismo, entre los que se encuentran: su variada flora y fauna, sitios históricos, arqueológicos, sistemas cavernarios y eventos culturales; además cuenta con esenciales vistas como es el mirador natural desde donde se puede observar la ciudad y la bahía.

Situación actual del spl agropecuario del municipio gibara 
La agricultura, ganadería, caza, pesca y silvicultura es el segundo sector en importancia para el municipio y de igual manera desde el municipio para la provincia, pero para analizar el comportamiento del sector en Gibara, es importante tener presente algunos de los rasgos estructurales que identifican hoy el comportamiento del sector. Este adquiere tal importancia a partir de la División Político Administrativa realizada en 1975 en que Velasco, antes municipio, pasa a ser un Consejo Popular del municipio Gibara, por lo que este de ser un municipio con una fuerte tradición en la pesca como actividad fundamental, pasa a ser la agrícola la fundamental y desde el 2008 se comienzan a desarrollar inversiones a partir del Programa para el Desarrollo de Gibara como municipio Turístico, dando lugar a su declaración en la Feria de Turismo de 2017 como destino turístico de Cuba y aunque en la actualidad los flujos turísticos han ido en aumento no se ha llegado a los resultados esperados a pesar de los atractivos con que cuenta.

Tanto la agricultura como el turismo y las fuentes renovables de energía; fueron identificados entre los sectores estratégicos del Plan de la Nación hasta el 2030 y se enmarcan entre los ejes de desarrollo de la Estrategia de Desarrollo Municipal de Gibara, por ello el grupo de DL del municipio seleccionó el sector de la agricultura para el estudio ya que juega un papel fundamental en el abastecimiento al sector del turismo del municipio; como proveedor de materia prima a otras entidades y en la satisfacción de las necesidades de la población así como, sus posibilidades para la transformación productiva de la estructura económica del municipio y la generación de productos para la sustitución de importaciones e incluso rubros exportables, por lo que se diagnosticó a partir de los subsectores presentes en el municipio.

En la actividad agropecuaria el mayor peso lo tiene el Sector Cooperativo como se muestra en la Tabla 1 y la entidad encargada de aunar, orientar y controlar el sector en el municipio es la Empresa Agroindustrial de Granos Gibara situada en la carretera HolguínGibara Km 17,5 en el Consejo Popular Floro Pérez estando entre sus principales producciones: las viandas, granos, hortalizas, frutas, leche y carne de ganado vacuno. Estas actividades productivas se desarrollan fundamentalmente en los Consejos Populares de Velasco I y II, Uñas, Floro Pérez y Arroyo Seco.

Tabla 1. Cantidad de formas productivas, áreas y su fuerza de trabajo.

\begin{tabular}{rlllcr}
\hline $\begin{array}{c}\text { Formas } \\
\text { productivas }\end{array}$ & Cantidad & $\begin{array}{l}\text { Área total } \\
\text { (ha) }\end{array}$ & $\begin{array}{c}\text { Fuerza de } \\
\text { trabajo }\end{array}$ & Hombres & Mujeres \\
\hline Ubpc & 8 & 4383.21 & 200 & 160 & 40 \\
Cpa & 17 & 19013.1 & 340 & 270 & 70 \\
Ces & 49 & 3459.4 & 4600 & 3400 & 1200 \\
Ueb & 2 & 248.7 & 120 & 85 & 35 \\
Total & 76 & 27104.41 & 5260 & 3915 & 1345 \\
\hline
\end{tabular}

Fuente: Empresa Agroindustrial de Granos Gibara, 2019 
Según el balance de la tierra realizado por GEOCUBA, la superficie agrícola del municipio es de 29806.85 ha, de esta superficie se encuentran cultivadas 19098.87 y sin cultivar el 10707.98 ha (pasto natural y tierras ociosas); con 3 formas productivas que comercializan directamente con las entidades hoteleras del municipio y junto a ello la Unidad Empresarial de Base (UEB) silvícola de Gibara que exporta anualmente 70 tn de carbón, una de sus principales producciones, así como aserrar la madera a la población, contando además con un total de 18 minindustrias de las cuales 16 se dedican a procesar condimentos, 1 productos encurtidos y 1 a la producción de pulpas de frutas, una planta de secado de granos, así como una UEB dedicada al procesamiento de carnes para la producción de embutidos con destino principalmente a los mercados turísticos.

El municipio cuenta con una gran diversidad de productos en los cultivos varios; con áreas de cultivos que presentan buenos rendimientos, aunque el déficit de sistemas de riego que sólo cubren 4811,2 ha (17,75\%) de la superficie agrícola; incide de manera negativa en la explotación eficiente del potencial con que cuenta, como se muestra en la

Tabla 2. Producciones de cultivos varios del municipio

\begin{tabular}{lllll}
\hline \multicolumn{1}{c}{ Viandas } & Hortalizas & Leguminosas & Frutas & Cítricos \\
\hline Boniato & Tomate & F. Negros & Coco & Naranja \\
Yuca & Cebolla & F. Colorados & Mango & Limón \\
Name & Ajo & F. Carita & Guayaba & Toronja \\
Malanga & Pimiento & Garbanzo & F. Bomba & \\
P. Fruta & Calabaza & Soya & Piña & \\
P. Vianda & Pepino & Maní & Melón & \\
P. Burro & Col & & Mango & \\
\hline
\end{tabular}

Fuente: Empresa Agroindustrial de Granos Gibara, 2019

Por su parte el desarrollo de la ganadería de forma general se ve afectado por los factores climatológicos; las producciones de ganado menor son mínimas, y no se aprovecha la ventaja de que estos son más resistentes a la sequía, sobre todo el caprino. De igual manera se desaprovechan las condiciones idóneas con que cuenta el municipio para el desarrollo de la pesca a pesar de contar con condiciones para ello.

Tabla 3. Composición de las producciones pecuarias

\begin{tabular}{lllll}
\hline Carne vacuna & Carne aves & Carne ovina & Pescado & Huevos \\
Carne porcina & Carne cunícula & Carne caprina & Miel de abeja & Leche
\end{tabular}

Fuente: Empresa Agroindustrial de Granos Gibara, 2019

De forma general el municipio cuenta con una gran diversidad de productos como base para la exportación y sustitución de importaciones entre los que destacan: 
- Granos y cereales; tubérculos, raíces y otras viandas; hortalizas y otros vegetales en estado natural o procesada; frutas naturales y procesadas; condimentos frescos o secos; flores, posturas de plantas frutales, forestales y ornamentales; plantas condimentosas, medicinales frescas o secas; semillas botánicas que incluyen plántulas y posturas de hortalizas; posturas de tabaco; caña de azúcar para alimento animal; productos no madereros del bosque entre ellos guano, palmiche, yaguas, resinas, bambú y semillas; madera en bolo, rolliza, aserrada, cuje para tabaco y cobijas, postes vivos, postes y traviesas; materia orgánica, humus de lombriz; subproductos de cosechas para alimento animal; forraje verde o seco; aceite de ajonjolí, de maní, de girasol, de palma de corojo y de otras plantas oleaginosas; animales vivos de ganado mayor y menor; carnes de ganado mayor y menor, en estado natural y procesada; cuero fresco, presalado, curtido y natural de ganado mayor y menor; huesos y vísceras; leche de ganado mayor y menor; carbón vegetal; leña para combustible; animales comerciales de trabajo; animales comerciales de ganado mayor en pie o en bandas a la industria cárnica; ganado genético y pie de cría de aves rústicas y sus huevos.

Entorno institucional. (instituciones de regulación, de apoyo material y financiero, de servicios, de innovación y capacitación)

La institución de regulación a nivel de provincia y país es el Ministerio de la Agricultura (MINAG), de apoyo material y financiero; Delegación Municipal de la Agricultura y el Banco de Créditos y Comercio (BANDEC), Grupo Empresarial de Logística Ministerio de la Agricultura (GELMA); de innovación y capacitación la Unidad Estratégica de Investigación y Capacitación de la Agricultura en Holguín (UEICAH) y el Centro Universitario Municipal, además cuentan con un alto potencial científico en la Empresa entre ellos Ingenieros Agrónomos, Veterinarios, Economistas, etc.

Instituciones que son poco aprovechadas por el sector en lo fundamental las orientadas a la innovación y la capacitación debido a la ausencia de relaciones formalizadas entre estas y la falta de visión del papel de la ciencia en los procesos productivos que impliquen la agregación de valor a productos de la actividad agropecuaria, la silvicultura y la pesca y para el desarrollo de la agroindustria como actividad económica que comprende la producción industrial y comercialización de productos agropecuarios, forestales y otros recursos naturales.

\section{Mercado meta que se propone alcanzar}

En las proyecciones del municipio, los principales mercados que la empresa desea abastecer de forma regular y segura son: el polo turístico de Guardalavaca, los Mercados agropecuarios del municipio y el municipio cabecera, así como las ferias, pues aún las producciones son insuficientes para cubrir estas demandas. Entre sus principales clientes se encuentran: Combinado Lácteo, Combinado Cárnico, Planta Comercializadora y Frutas Selectas; para las cuales la Empresa Agroindustrial tiene un plan de entrega que se encuentra por debajo de las demandas actuales para la producción de productos terminados. 


\section{Principales productos que sustituyen importaciones o posibles exportaciones}

Entre los principales productos que en la actualidad se tienen identificados en la empresa para este fin se encuentran: el maíz, tomate, frijol, carne de res y miel de abeja, aunque desde el punto de vista de los autores, se le debe prestar atención a aquellos productos que son demandados por los polos turísticos, ya que son considerados como productos de exportación o que sustituyen importaciones, en el caso del Complejo Turístico Iberostar Gibara se determinaron las cantidades y variedades de productos que se consumen y que pueden ser producidas en el municipio. Se tuvo en cuenta las temporadas de mayor y menor afluencia de clientes, así como las cosechas; factores que provocan que la cantidad y variedad de los productos varíe como se muestra en el Anexo 2 Tabla 4

\section{Valoración general de los problemas que afectan el spl agropecuario}

Para la determinación y valoración de los principales problemas que afectan al sector agropecuario del municipio se realizaron entrevistas a directivos vinculados con estas actividades, los cuales aportaron las siguientes opiniones:

- escasa base alimentaria para los animales,

- altas temperaturas y bajas precipitaciones; Baja natalidad animal,

- fluctuación de la fuerza de trabajo; Faltan piezas de repuestos para las maquinarias,

- atraso en la inversión de la Conductora del Trasvase,

- no se aprovecha el potencial de la pesca

Mediante entrevistas se pudo conocer que estos problemas tienen su origen en lo fundamental por la influencia de factores económicos, políticos y naturales internos y externos a los que se enfrenta el país y por tanto el municipio, lo cual limita la entrada de recursos al sector tanto financieros como materiales, provocando roturas reiteradas en los medios de producción, así como obsolescencia de las maquinarias e instalaciones, lo que retrasa o pospone las inversiones ocasionando el éxodo de trabajadores hacia otros sectores mejor remunerados y con mejores condiciones de trabajo, por otra parte, la mayor e importantes partes de las actividades productivas del sector se realizan a cielo abierto, lo que provoca dependencia de los factores climatológicos, afectando tanto los cultivos como a la ganadería.

\section{Principales fortalezas, debilidades, oportunidades y amenazas que presenta el spl}

El diagnóstico realizado al SPL escogido permitió identificar las siguientes:

\section{Fortalezas}

Gran número de minindustrias; Trabajadores con experiencias en sus actividades; Potencial científico en su capital humano; Aprovechamiento de la silvicultura; Potencial para el desarrollo del sector; Municipio especializado en sectores de rápido crecimiento; Desarrollo del turismo; Potencial para la utilización de servicios y recursos de otras industrias; Sus pobladores.

\section{Debilidades}


Áreas con sistemas de riego; Base alimentaria para los animales; Fluctuación de la fuerza de trabajo; Suelos ociosos con condiciones agropecuarias; Erosión de los suelos; Capacitación de los productores en los temas sobre SPL y CP; Aprovechamiento de las potencialidades pesqueras; Baja natalidad de los animales; Relaciones de articulación entre productores, industrias y mercados; Proyectos de DL con enfoque de CP; Aprovechamiento del potencial científico que posee el territorio; Competitividad territorial; Rehabilitación de los suelos.

\section{Oportunidades}

Potencial de nuevos productos para la exportación; Declaración de Gibara como destino turístico de Cuba; Hermanamiento de Gibara con el municipio Español Prat de Llobregat; Es un municipio PADIT; Demanda de productos agropecuarios; Interés de la alta dirección del país y la provincia en el desarrollo de sectores claves de la economía del municipio (Agricultura, Materiales de la Construcción, Energía Renovable y Turismo); Desarrollo de eventos Internacionales en el municipio; Potencialidades en el territorio para las inversiones

\section{Amenazas}

Situación climatológica; Atraso en la inversión en la Conductora Trasvase; Limitaciones de recursos materiales, financieros y técnicos; Drenaje y erosión de los suelos; Situación internacional inestable y agravada; Iniciativa de los organismos vinculados al sector para aprovechar sus potencialidades; Insuficiente información confiable, pertinente y consistente para la realización del diagnóstico; División político administrativa.

Mediante trabajo en grupo, de los resultados del diagnóstico se elaboró la Matriz de Evaluación de Factores Internos (MEFI-Tabla 5) cuyo análisis arrojó un resultado total ponderado (RTP) de 2,45 mostrando un predominio de las debilidades sobre las fortalezas denotando que la situación interna del sistema es desfavorable influyendo en un mayor grado sobre los resultados del sistema; mientras que desde el punto de vista externo el resultado total ponderado que arroja la Matriz de Evaluación de Factores Eternos (MEFETabla 6) fue de 267 lo que evidencia un predominio de las oportunidades sobre las amenazas lo que expresa que el sistema presenta una situación externa favorable, lo que indica que el SPL Agropecuario de Gibara tiene que con las oportunidades que le brinda el entorno formular estrategias y acciones que le permitan eliminar las debilidades y atenuar el efecto de las amenazas del entorno por lo que su estrategia de desarrollo hasta el 2030, ha de orientarse a estrategias de tipo Adaptativas a partir de su posición estratégica interna y externa: 
Cuadro1. Resultados de la Matriz DAFO

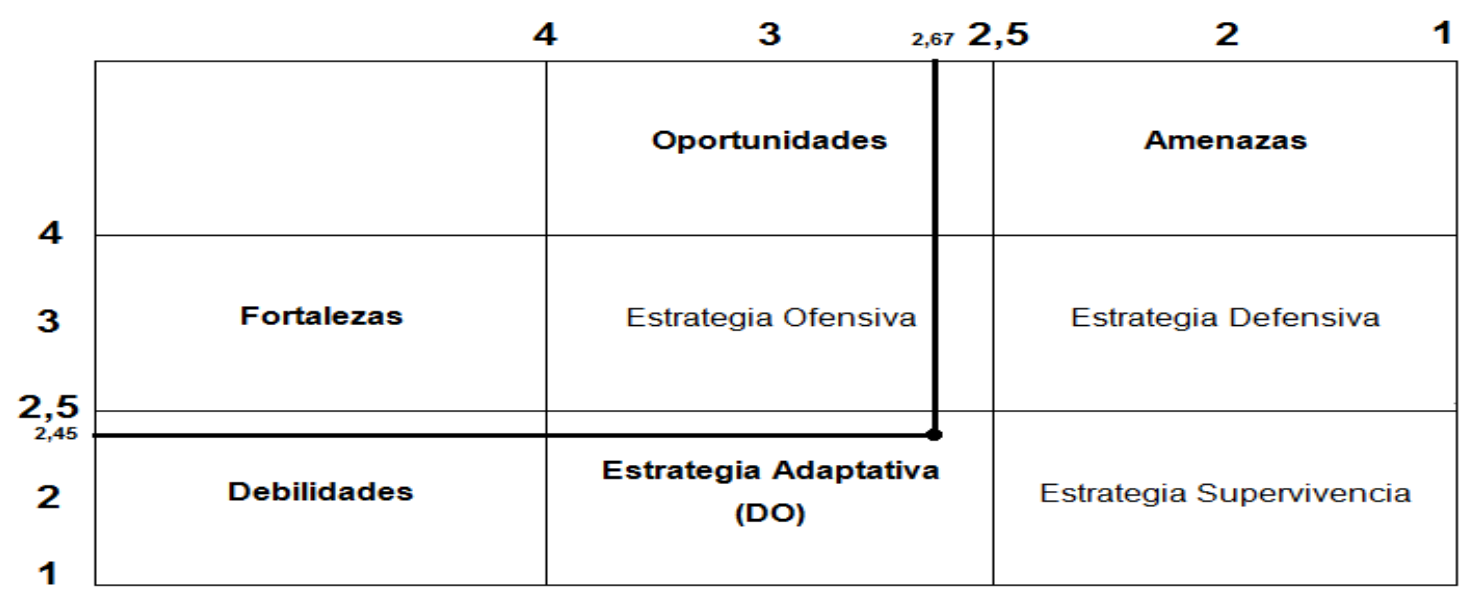

Fuente: elaboración propia

Por lo que entre las estrategias a seguir en torno al fortalecimiento del SPL debe partir de tener en cuenta las:

\section{Cadenas productivas que tienen como eje primario la producción agropecuaria}

Las CP no son estructuras que se construyen desde el Estado, ellas existen desde hace mucho tiempo y siempre existirán de manera formal o informal; de ahí que por su contribución al desarrollo desde lo local, y por su contribución a la elevación de la calidad de vida de la población en el Plan de la Nación hasta el 2030 la producción de alimentos se encuentren entre los sectores estratégicos identificados para el futuro de la nación, por lo que no se pueden considerar como elementos separados del resto de la economía del país, sin embargo en la actualidad no existen políticas públicas que institucionalicen las $\mathrm{CP}$ en los territorios, sin embargo la voluntad política del país es que las mismas sean institucionalizadas a partir de identificación, diseño y completamiento de proyectos de DL que cierren las brechas en las relaciones de articulación.

Las investigaciones realizadas con anterioridad en el municipio Gibara y el diagnóstico del SPL Agrícola permitió identificar $31 \mathrm{CP}$ en el sector y los subsectores objeto de estudio en estado incipiente al no estar la mayoría formalmente establecidas, en estas el cultivo de cada producto agrario y pecuario primario es óptimo por razones de vocación productiva de los suelos tanto para la alimentación humana como de los animales por lo que se pudieron definir oportunidades de inversión en estas, entre ellas se identificaron:

\section{Subsector de la agricultura}

1. CP Derivadas de los granos:

Cadenas productivas de cultivo del frijol: F. Negros, F. Colorados, F. Carita, Garbanzo, Soya.

Cadena productiva de cultivo y procesamiento del Maíz.

\section{CP Derivadas de las hortalizas:}

Cadenas productivas de cultivo y procesamiento de hortalizas: Tomate, Cebolla, Ajo, Pimiento, Calabaza, Pepino, Col. 


\section{CP Derivadas de las frutas:}

Cadenas productivas de cultivo y procesamiento frutas: Coco, Mango, Guayaba, F. Bomba, Piña, Melón.

\section{Subsector de ganadería}

\section{CP Derivadas de la ganadería:}

Cadenas productivas de la producción y procesamiento de carne animal: Ganado vacuno, Ovino, Caprino, Porcino, Avícola y Cunícula (conejo).

\section{Subsector de silvicultura}

\section{CP Derivadas de la silvicultura:}

Cadenas productivas de la producción de: Flores, Plantas ornamentales, Posturas de árboles frutales, Posturas de árboles maderables y carbón vegetal.

\section{El ejemplo de la cadena productiva de la guayaba}

El impulso a esta cadena lo generó el equipo de dirección de la Unidad Empresarial de Base para la Producción y Comercialización de Conservas de Frutas y Vegetales Turquino de Holguín y en coordinación con el grupo de desarrollo Local se trabaja en su consolidación; esta empresa fue fundada antes del triunfo de la revolución en 1943; el 15 de diciembre de 1976 se ratifica su creación como empresa y en mayo del 2011 se crea la Empresa de Conservas de Vegetales con personalidad jurídica propia a la que quedó integrada la Unidad Empresarial de Base Turquino en Holguín; la misma a partir de las potencialidades identificadas entre las que se destacan: la incorporación a la cartera de la empresa de nuevas producciones; capacidad para elevar la producción de guayaba a 1000 tn de productos terminados con la tecnología actual; capacidad de incremento de la materia prima- pulpa de guayaba semielaborada a partir del encadenamiento; posibilidad de fomento de otras cadenas productivas: Maní, Fruta Bomba, Mango, Tomate y Vegetales; apertura de firma de contratos de cooperación y suministro con las diferentes formas productivas.

\section{Antecedentes de la cadena productiva de la guayaba}

Hasta el 2017 las producciones de guayaba en el municipio iban hacia diferentes destinos, en lo fundamental el mercado informal, por lo que durante 12 años la fábrica se mantuvo sin entrada significativa de esta materia prima, hasta ese año la UEB mantenía contrato con Acopio con producciones insuficientes que permitiera aprovechar la capacidad instalada en fábrica, por lo que se traía de otras provincias como Matanzas (Jagüey Grande) y Villa Clara con los correspondientes costos y gastos que ello entrañaba; en 2018 resultado de estudios realizados se comienzan a establecer relaciones de articulación con productores (municipio Gibara con 1 y luego se incorpora Calixto García con 2) que contaban con minindustrias transformadoras y se inicia la Cadena Productiva de la Guayaba, produciendo en ese año 500 tn de productos terminados a partir de la pulpa de guayaba semielaborada.

\section{La guayaba- generalidades}


Las guayabas (Psidium) son un género de unas 100 especies de árboles tropicales y árboles pequeños en la familia Myrtaceae, nativas del Caribe y América Central, del Sur y el Norte; es rica en vitaminas A, B, C siendo entre las frutas una de las que mayores niveles tiene de vitamina $C$ por gramo por lo que es un antigripal natural; también es usada extensivamente en dulces, jaleas, mermeladas y jugos.

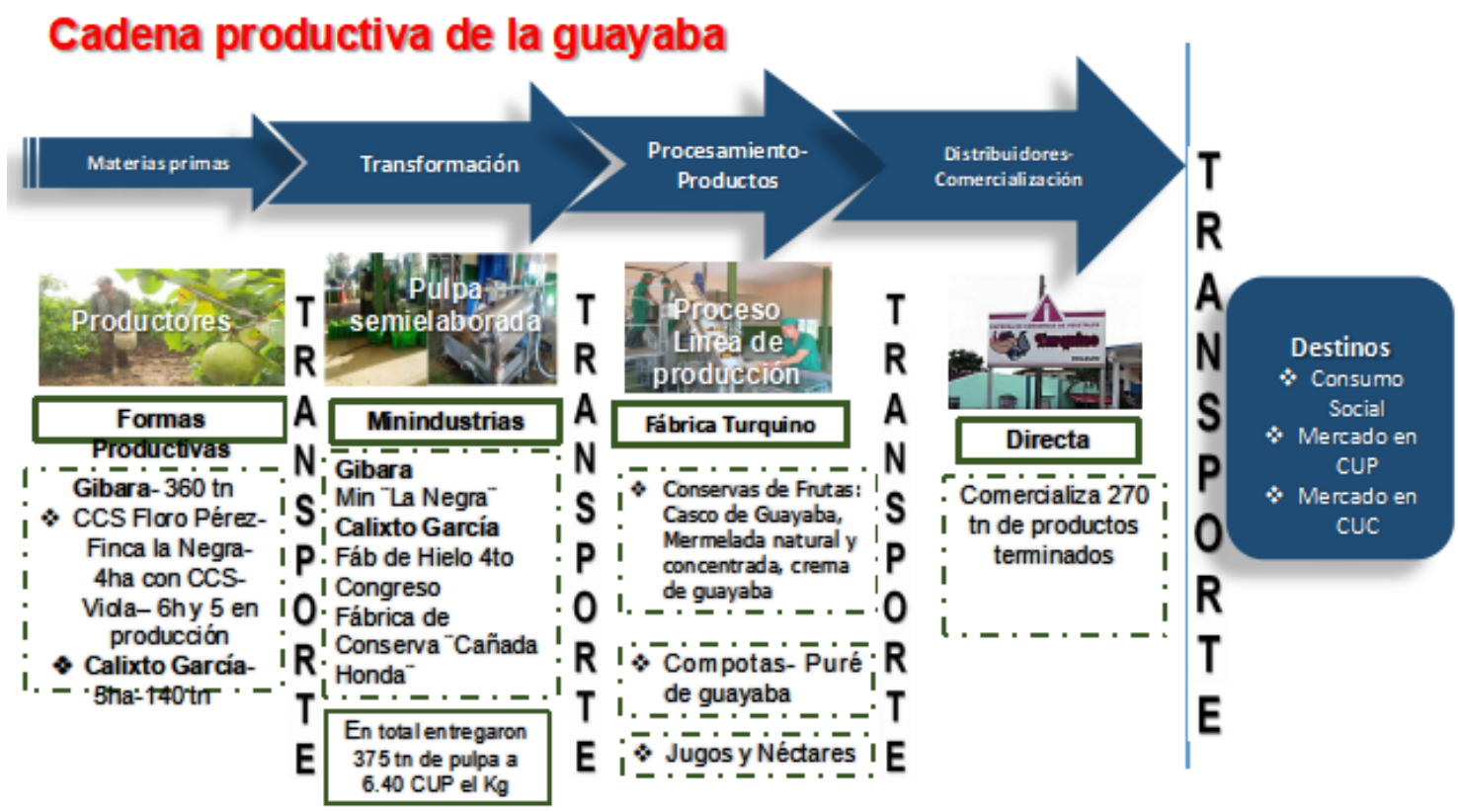

Fuente: elaboración propia a partir de los resultados del análisis

El trabajo realizado ha traído como resultado a la UEB Turquino, hasta la fecha: el desarrollo de capacidades para el aseguramiento de los envases; la factibilidad de los precios, que son atractivos para los productores (6,40 CUP el kg de pulpa semielaborada); el suministro del aditamento tecnológico para las minindustrias que permitió lograr el llenado aséptico de la materia prima, lo que trajo consigo una mayor durabilidad, mejor conservación e inocuidad de la misma; los ingresos a las minindustrias fueron superiores a 1,8 millones de pesos lo que les permitió a estas invertir un porciento en la compra de plantaciones en la provincia Ciego de Ávila ascendentes a 40 mil plantas y 10 hectáreas sembradas, con un valor ascendente a 400 mil pesos por este concepto por lo que, a partir de la diversidad de las plantas y los sis temas de clonación, a través de injertos y esquejes, ya comienzan a despuntar las posturas de las futuras plantaciones de guayaba; el Ministerio de la Agricultura amplió las capacidades de siembra para algunos productores asociados a cooperativas de frutales con 10 hectáreas logrando un rendimiento de $750 \mathrm{Kg}$ de pulpa por tonelada de guayaba, lo que convertido en producción terminada, representa 1 tonelada de productos terminados.

El procedimiento empleado se aplicó en Consejos Populares de los municipios Banes, Antilla y Holguín con resultados que permitieron la identificación de SPL y encadenamientos productivos que sirven de base a la actualización de la Estrategia de Desarrollo Municipal, así como generar nuevas relaciones de articulación con la industria, posibilitando el aprovechamiento de las capacidades instaladas. 


\section{Conclusiones}

- El análisis y síntesis de la literatura nacional e internacional permitió confeccionar el marco teórico-referencial de la investigación en las que se reconoce la importancia de los SPL para la trasformación de la estructura económica territorial y el desarrollo territorial, sin embargo en las condiciones de la economía cubana en general y del municipio en particular es insuficiente su desarrollo, aplicación y normalización por los gobiernos locales que permita aprovechar el potencial endógeno con que cuentan.

- Los resultados del presente trabajo tienen su base en diagnósticos realizados en el 2016 en los municipios de la provincia Holguín, y con el fin de contribuir a convertir el municipio Gibara en municipio turístico, se aplicó en el 2018-19 la Etapa 3, pasos 3 y 4 del procedimiento, lo que permitió diagnosticar el SPL agropecuario en el municipio y su contribución a la sustitución de importaciones con base en el sector del turismo como sector estratégico en el municipio.

- El SPL diagnosticado permitió que se identificaran 31 cadenas productivas que tienen con eje primario en estado incipiente el sector de la agricultura, caza, ganadería y silvicultura y la selección para su desarrollo de la cadena productiva de la Guayaba y otras que están en proceso de formalización (tomate, maní, fruta bomba), que permita en el mediano y largo plazo convertirlos en productos para la exportación o de sustitución de importaciones a partir de que el desarrollo del SPLA y las CP como línea estratégica de la Estrategia de Desarrollo Municipal.

\section{Referencias bibliográficas}

Aznar Sánchez, J. Á. (2011). Análisis sobre Sistemas Productivos Locales Agroindustriales. Cuadernos de estudios agroalimentarios, 07-10.

Consejo de Administración Provincial. Informe de Desarrollo local y su impacto en la provincia Holguín. (2018).

Madruga Torres, A. G. (2014). Los sistemas productivos locales en la gestión del desarrollo local en Cuba. Ciencias Holguín, XX (3), 1-12.

Madruga Torres, A. G., Rojas Riverón, M., Clarke Bloomfield, M., \& Sarmiento Rodríguez, Y. (2015). La Cadena de Valor y el Desarrollo Local. Razones para una propuesta de proyecto en la producción acuícola de Mayarí. Universidad de Holguín, sede "Oscar Lucero Moya".

Mayán Sánchez, A. (2017). Diagnóstico prospectivo estratégico del municipio Gibara. Tesis en opción al título de Licenciada en Economía, Universidad de Holguín, sede Oscar Lucero Moya

Naclerio, A. (2008). Sistemas Productivos Locales: Aspectos conceptuales. In M. d. E. Producción (Ed.). 
Naclerio, A. (2010). Sistemas Productivos Locales, Políticas Públicas Y Desarrollo Económico. In B. Aires (Ed.).

Organización de Naciones Unidas. Transformar nuestro mundo: La Agenda 2030 para el Desarrollo Sostenible. (2015) (pp. 1-40).

Partido Comunista de Cuba. (2017). Lineamientos de la Política Económica Y Social del PCC y la Revolución para el Período 2016-2021. Presentado en VII Congreso del PCC. La Habana: Editora Política. P. 6 y 29.

(2017). Plan Nacional de Desarrollo Económico y Social hasta el 2030: Visión de la Nación, Ejes y Sectores Estratégicos. La Habana: Editora Política, p.5-25.

Rendón Acevedo, J. A., \& Forero Muñoz, J. D. (2014). Sistemas Productivos Locales: Estrategias Empresariales para el Desarrollo Semestre Económico, 17(35), 75-94.

Alburquerque, F. (1999). Servicios empresariales y desarrollo económico local: una reseña temática. (2004). El Enfoque del Desarrollo Económico Local. In O. I. d. Trabajo (Ed.).

Acevedo, J., Gómez, M., y López, T. (2012). Análisis de la cadena de valor hortofrutícola del municipio Marianao en La Habana, Cuba. Ingeniería Industrial/ISSN18155936, (XXXIII).

Bateman, A. (2016). Las Políticas Urbanas para el Aprovechamiento de Las Economías de Aglomeración y la Inclusión Productiva.

Cárdenas, A. (2015). Encadenamientos Productivos: La Guía Práctica. Bogotá: Colegio de Estudios Superiores de Administración-CESA, Administración de Empresas.

Consejo de Administración Provincial. Visita de Trabajo al municipio Gibara. (2019).

Fundación CODESPA. (2011). Metodología de análisis de cadenas productivas bajo el enfoque de cadenas de valor.

Giner Pérez, J. M., María Beneyto, J. S., \& Fuster Olivares, A. (2006). Los Sistemas Productivos Locales en la Comunidad de Valeciana. In U. d. Alicante (Ed.), (pp. 109-126).

Heyden, D. v. d., \& Camacho, P. (2004). Guía Metodológica para el Análisis de Cadenas Productivas. In C. I. d. C. p. e. D. Agrícola (Ed.). 
Productivas. In P. RURALTER (Ed.).

Hernández, R. (2018). Minindustrias favorecen encadenamientos productivos. 7 de junio. Granma. Recuperado de www.granma.cu

Hirschman, A. (1958). La estrategia del desarrollo económico. Fondo de Cultura Económica, México.

Iglesias, D. (2002). Cadenas de valor como estrategia: las cadenas de valor en el sector agroalimentario, 5-7. Recuperado de http://inta.gob.ar/sites/default/files/scripttmp-cadenasdevalor.pdf.

Isaza, J. (2008). Cadenas productivas. Enfoques y precisiones conceptuales, 9-25. Recuperado de http://revistas.vexternado.edu.co/index.php./sotavento/article/view/1602

Madruga Torres, A. G. (2015). Aglomeraciones Productivas como Base para Los Sistemas Productivos Locales. Universidad De Camagüey Ignacio Agramonte Loynaz.

Madruga Torres, A. G., \& González Fontes, R. (2011). Diagnóstico de la estructura productiva de los territorios para generar alternativas que favorezcan encadenamientos productivos en las aglomeraciones. In U. d. H. O. L. Moya (Ed.).

Oficina Nacional de Estadística e información. (2017). Anuario Estadístico de Holguín. Edición 2018.

Partido Comunista de Cuba. (2017). Bases del Plan Nacional de Desarrollo Económico y Social hasta 2030: Propuesta de Visión de la Nación, Ejes y Sectores Estratégicos, Tabloide de la UEB Gráfica Empresa de Periódicos.

(2017). Lineamientos de la Política Económica Y Social del Partido y la Revolución para el período 2016-2021. Tabloide de la UEB Gráfica Empresa de Periódicos.

Ramos Acero, J. C., \& Solano Rincón, J. E. (2013). Eslabonamientos Productivos un Análisis de Características y Estructura Económica Regional en Colombia In E. C. D. I. J. GARAVITO (Ed.).

Vergaray, P. (2013). Cadenas Productivas, Una mirada panorámica a CAMPOSOL.

Villacorta R., I., Quiroga A., J. C., \& Zubieta, J. (2006). Guía para la Elaboración de Estudios de Cadenas Productivas Locales. 


\section{PARA CITAR EL ARTÍCULO INDEXADO.}

Clarke Bloomfield, M., Madruga Torres, A. G., Rodríguez Galindo, C. R., \& Carballosa Ramírez, J. L. (2021). Sistemas productivos locales y encadenamientos productivos a favor del desarrollo local: El sistema productivo local agropecuario del destino turístico Gibara en holguín. Explorador Digital, 5(1), 437-457. https://doi.org/10.33262/exploradordigital.v5i1.1512

\section{¿Ciencia}

El artículo que se publica es de exclusiva responsabilidad de los autores y no necesariamente reflejan el pensamiento de la Revista Explorador Digital.

El artículo queda en propiedad de la revista y, por tanto, su publicación parcial y/o total en otro medio tiene que ser autorizado por el director de la Revista Explorador Digital.
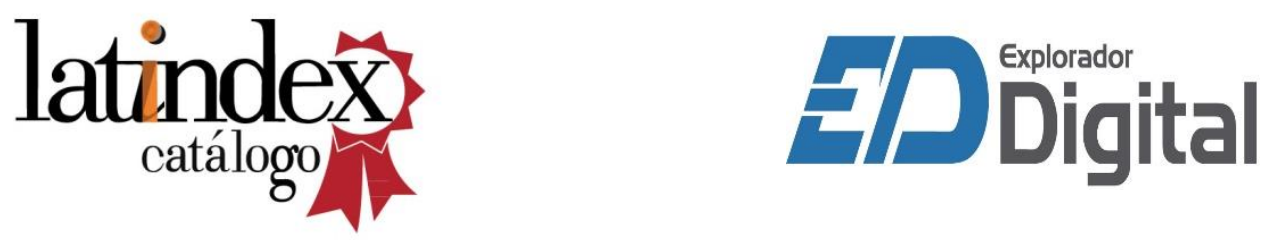